\title{
Possible Method of Memorizing Tonal Sound Signal Frequency in the Human Auditory System
}

\author{
Margarita Stefanovich \\ Independent Researcher, Saint-Petersburg, Russia
}

Email address:

marg.stefanovich@yandex.ru

\section{To cite this article:}

Margarita Stefanovich. Possible Method of Memorizing Tonal Sound Signal Frequency in the Human Auditory System. Advances in Applied Physiology. Vol. 4, No. 2, 2019, pp. 19-27. doi: 10.11648/j.aap.20190402.12

Received: December 18, 2019; Accepted: January 15, 2020; Published: February 14, 2020

\begin{abstract}
The book "Das Ohr als Nachrichten Empfänger" by Zwicker and Feldkeller was published in 1967. It four experiments researching into the study of human acoustic sensation in the perception of a tonal sound signal. Comprehensive analysis of their results allows us to understand that the auditory system remembers information regarding the frequency of sound on the basis of on the principle of place. According to their biophysical characteristics the complex of elements Inner Hair Cells-Basilar membrane-Outer Hair Cells (OHC-BM-IHC) is a series connection of parallel resonant circuits. During the process of perceiving a tonal sound signal, the greatest amplitude of the transverse oscillations of the BM of the cochlea is observed at that point of the basilar membrane where the signal frequency is closest to the resonance frequency of the contour. The auditory system stores information about the frequency of the sound through the sequence number of the IHC, located in the middle of the general group of cells with an elevated receptor potential (RP). The projection of IHC can be considered as a measuring scale with digitized points. Neurons with excitatory and inhibitory inputs function as logic chips in a computer. The neural network stores information about the audible pitch as a number in binary code in a group of ordered memory neurons. The understanding of main principles of functioning of human auditory system is necessary for improvement of quality of speech perception through Cochlear Implant.
\end{abstract}

Keywords: Auditory System, Frequency of Sound Signal, Audible Pitch

\section{Introduction}

At the International Symposium on Hearing (ISH), which was held in 2018 in Snekkersten, Denmark, the special peculiarities of auditory sensations in the perception of complex (multi-frequency) audio signals and possible ways of helping people with hearing loss [1] were among the main topics of discussion. However, possible ways of encoding information about the sound signal frequency according to the principle of place or the temporal principle were barely discussed. Most specialists in psychoacoustics deny the possibility of implementing the real frequency resolution of human hearing while encoding information using the principle of place. They assume that the auditory system encodes the frequency information in the frequency range up to $4 \mathrm{kHz}$ according to the temporal principle, and uses the principle of place in the higher frequency range [2]. In order to study auditory sensations, the simplest tonal signals have to be used while conducting experiments. Any compound signal in the complex of OHC-BM-IHC elements is divided into separate frequency components. The auditory system stores information about the frequency of each individual component.

The book "Das Ohr als Nachrichtenempfänger" by Zwicker, Feldkeller [3] was published in 1967. It contains a description of four experiments to study auditory sensations in the perception of tonal sound signals. A comprehensive analysis of the results of the experiments suggests that there is a possible algorithm for storing information about the frequency of the sound with real difference limens for frequency (DLFs). Of the known cochlea models, the Bollerott model [4] is the closest to the frequency information memorization algorithm proposed in this article. The block diagram of the model shows that the ends of the auditory nerve fibers, innervating one IHC, are combined on the synapses of a single summing auditory neuron (SAN) at the input of the first projection of the cochlea frequency scale (PCFS). This means that the discharge rate of the SAN is 
proportional to the receptor potential (RP) of the innervated IHC.

While describing the block diagram of the model, the author notes that the quantity of band-pass filters (BF) in other cochlea models may range from 24 to 112 and even up to 480 . Based on an analysis of the results of the known experiments, this article considers the possible algorithm for coding information about signal frequency according to the principle of place. It also describes a possible way of normalizing the displacement of the IHC group with an increased RP along the BM depending on the frequency of the audible tone and a possible way of extracting a group of 146 cells located symmetrically relative to the central IHC from the general IHC group with an elevated RP. A parallel analysis of physiological experiments to study the frequency scale of the guinea-pig cochlea and human cochlea has been carried out in order to confirm the reliability of the results of Zwicker and Feldkeller's experiments, on the basis of which a possible algorithm for storing information about the frequency of sound can be built $[5,6]$.

In the scientific literature on sensory systems and neurophysiology, the question of what the brain actually remembers when it perceives sensory stimuli is not discussed at all. The auditory system has been studied in detail. Psychoacoustics experts until the $1980 \mathrm{~s}$ tried to find an answer to the question: "Why is the audible pitch is not proportional to the frequency of the sound signal?" Numerous experiments were conducted. Very close to the answer to this question came Zwicker and Feldkeller [3], but they didn't do one last step. After 1990 the question of possible encoding of information about the frequency of an audio signal has not been discussed even in monographs on psychoacoustics.

\section{Method}

\subsection{The Correlation Between the Location of the BM Maximum Transverse Oscillations and the Frequency of the Audio Signal}

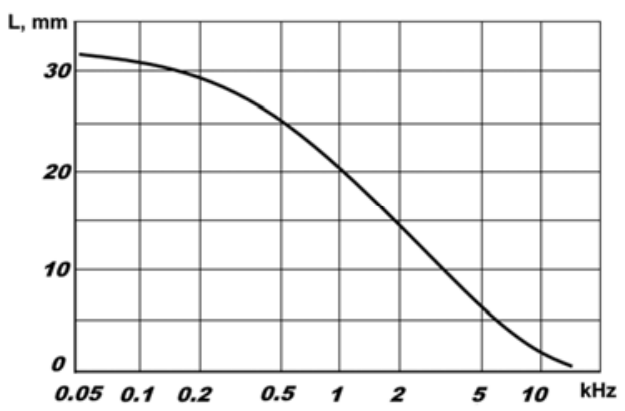

Figure 1. The correlation between the maximum transverse oscillations of the basilar membrane of the cochlea and the frequency of the tonal signal. The abscissa axis is the tone frequency in $\mathrm{kHz}$, the ordinate axis is the distance from the L base in $\mathrm{mm}[3]$.
The graph in Figure 1 is constructed from the averaged results of a physiological experiment studying the frequency response of the cochlea in 17 guinea-pigs under anesthesia and 8 people hospitalized after accidents $[4,5]$. The frequency characteristics of the guinea-pig and human cochlea are identical. All hearing system experts agree that when the frequency of the sound signal increases, the point on the BM with the highest amplitude of transverse oscillations (the other name for it is the position of maximum vibration of the basilar membrane or PMVM) really shifts to the base of the cochlea. However, no one had considered this graph as the true ratio between the frequency of sound and the distance from the point of the PMVM to the base of the cochlea. This graph is similar for all people with normal hearing. Most people with normal hearing can correctly reproduce the sound they hear with their voice. When hearing is impaired, the slope of the line of the graph in the frequency range in which the auditory thresholds change decreases. A person hears different sounds as equal in pitch. The graph in Figure 1 allows us to measure the distance from the main excitation point of the cochlea membrane (PMVM) to the "L base" for any frequency in "mm".

\subsection{The Results of the Experiment Measuring the Width of the Frequency Channels (FC)}

The frequency scale of human hearing is divided into 24 frequency channels. Each FC consists of 146 IHC. Zwicker and Feldkeller experimentally measured the width of all the $\mathrm{FC}$ and indicated the central and boundary frequencies for each FC [3]. Each FC consists of 146 IHC, which are located along the length of the $1.3 \mathrm{~mm}$ basilar membrane of the cochlea. There is a PMVM for a frequency of $50 \mathrm{~Hz}$ in the middle of FC1. For the $50 \mathrm{~Hz}$ frequency the linear coordinate PMVM is located at a distance of $0.65 \mathrm{~mm}$ from the apex of the cochlea (Lapex). The sequence number of the IHC in PMVM is $\mathrm{NO}=73$. The linear coordinate of the PMVM relative to the $\mathrm{L}$ apex for the center frequency of any FC can be calculated in "mm" and by the sequence number of IHC in PMVM (N0):

$$
\begin{gathered}
\mathrm{L} \text { apex }=[0.65+1.3 \times(\mathrm{Nfc}-1)] \mathrm{mm} \\
\mathrm{N} 0=[73+146 \times(\mathrm{Nfc}-1)]
\end{gathered}
$$

Here, L apex is the distance from the apex of the cochlea, $\mathrm{N} 0$ is the sequence number of the IHC in the PMVM, Nfc is the sequence number of the frequency channel. The sum of the numerical values of the linear coordinates of the PMVM for each frequency relative to the base of the cochlea and relative to apex of the cochlea ( $\mathrm{L}$ base $+\mathrm{L}$ apex) is equal to the total length of basilar membrane from the apex to the base. 
Table 1. The experimentally determined central, boundary frequencies and range for 24 FC [3].

\begin{tabular}{|c|c|c|c|c|c|c|c|c|c|c|c|}
\hline $\mathrm{N}$ fc & Fcent. & $\Delta \mathrm{F}$ fc & Fbound & $\mathrm{N}$ fc & Fcent. & $\Delta \mathrm{F}$ fc & Fbound & $\mathrm{N}$ fc & Fcent. & $\Delta \mathrm{F}$ fc & Fbound. \\
\hline 1 & 50 & 80 & 20 & 9 & 1000 & 160 & 920 & 17 & 3400 & 550 & 3150 \\
\hline 2 & 150 & 100 & 100 & 10 & 1170 & 190 & 1080 & 18 & 4000 & 700 & 3700 \\
\hline 4 & 350 & 100 & 300 & 12 & 1600 & 240 & 1480 & 20 & 5800 & 1100 & 5300 \\
\hline 5 & 450 & 110 & 400 & 13 & 1850 & 280 & 1720 & 21 & 7000 & 1300 & 6400 \\
\hline 6 & 570 & 120 & 510 & 14 & 2150 & 320 & 2000 & 22 & 8500 & 1800 & 7700 \\
\hline 8 & 840 & 150 & 770 & 16 & 2900 & 450 & 2700 & 24 & 13500 & 3500 & 12000 \\
\hline
\end{tabular}

Note: fc - frequency cannel; Nfc - number of FC; Fcent. - central F, Hz; $\Delta \mathrm{F}$ fc - range of FC, Hz; Fbound - min F, Hz.

Table 2. Linear coordinates of PMVM for several frequencies, measured relative to the L base of cochlea according to the diagram in Figure 1 with using a digital ruler, and the linear coordinates of the PMVM for the same frequencies relative to apex of the cochlea $L$ apex, calculated from the width of the frequency channels [3].

\begin{tabular}{|c|c|c|c|c|c|c|c|c|c|}
\hline $\mathbf{F , ~ k H z}$ & 0,1 & $\mathbf{0 , 2}$ & $\mathbf{0 , 5}$ & 1 & 2 & 5 & 8 & 10 & Comment \\
\hline L base, $\mathrm{mm}$ & 30,5 & 28,9 & 24,9 & 20,0 & 14,0 & 5,8 & 2,8 & 1,8 & Figure 1 \\
\hline $\mathrm{L}$ apex, $\mathrm{mm}$ & 1,3 & 2,6 & 6,4 & 11,1 & 16,9 & 23,7 & 27,0 & 28,2 & Table 1 \\
\hline $\mathrm{Lbm}, \mathrm{mm}$ & 31,8 & 31,5 & 31,3 & 31,1 & 30,9 & 29,5 & 29,8 & 30,0 & Lbm $=$ Lbase + Lapex \\
\hline
\end{tabular}

The sums of the linear coordinates PMVM relative to the apex of the cochlea and relative to the base of the cochlea in the frequency range from $100 \mathrm{~Hz}$ to $10 \mathrm{kHz}$ are almost the same (the greatest discrepancy of the results is $\pm 4 \%$ from the average value of $30.8 \mathrm{~mm}$ ). The agreement of the measurement results of the linear coordinates of the PMVM in the experiments according to Figure 1 and calculated from the measurement results of the width of the frequency channels in Table 1 can be considered as evidence of the reliability of the data obtained by the authors of the experiments. The fact that the experiments were carried out in completely different conditions is a cause of particular admiration. Physiological experiments were carried out on the "open cochlea", otherwise the length of the BM segment cannot be measured in $\mathrm{mm}$. Zwicker and Feldkeller conducted psychoacoustic experiments 30 years later, with the active participation of the subjects with normal hearing.

\subsection{The Correlation Between the Audible Pitch and the Frequency of the Tonal Sound Signal}

Audible pitch is a feeling. In order to be able to compare the audible pitch when perceiving tonal sound signals with different frequencies, the conventional unit of measurement " 1 mel" was adopted. In the frequency range from $100 \mathrm{~Hz}$ to $500 \mathrm{~Hz}$, the numerical values of the frequency in " $\mathrm{Hz}$ " and the audible pitch in "mel" are the same.

The sums of the linear coordinates PMVM relative to the apex of the cochlea and relative to the base of the cochlea in the frequency range from $100 \mathrm{~Hz}$ to $10 \mathrm{kHz}$ are almost the same (the greatest discrepancy of the results is $\pm 4 \%$ from the average value of $30.8 \mathrm{~mm}$ ). The agreement of the measurement results of the linear coordinates of the PMVM in the experiments according to Figure 1 and calculated from the measurement results of the width of the frequency channels in Table 1 can be considered as evidence of the reliability of the data obtained by the authors of the experiments.

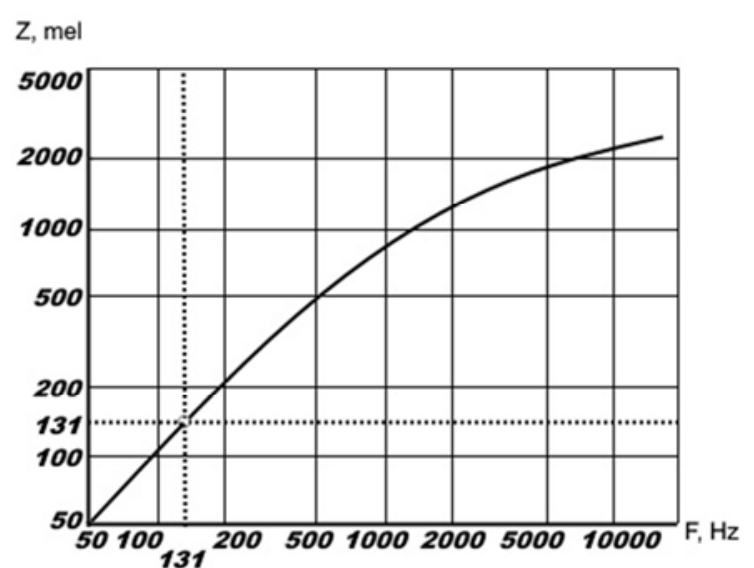

Figure 2. The correlation between the audible pitch $Z$, mel and the frequency $\mathrm{F}, \mathrm{Hz}[3]$.

In the experiment to measure "Dependence of audible pitch Z, mel on the frequency $\mathrm{F}$ in $\mathrm{Hz}$ ", the subjects compared the change in audible pitch with an increase in pitch frequency from $131 \mathrm{~Hz}$ to $165 \mathrm{~Hz}$ with the same change in audible pitch (by 1 third) during a consequent perception of two tones in the frequency range up to $8 \mathrm{kHz}$. A large group of subjects participated in the experiment. The averaged results for the entire group of subjects are shown in Figure 2. Other specialists in psychoacoustics, when conducting such an experiment, chose the starting frequency of $1000 \mathrm{~Hz}$ as the starting point [7]. In the course of the experiment, the initial frequency of $1000 \mathrm{~Hz}$ decreased successively by a third to $131 \mathrm{~Hz}$, and then after $1000 \mathrm{~Hz}$ it increased by a third to $8000 \mathrm{~Hz}$. As a result, a graph in the frequency range up to $500 \mathrm{~Hz}$ was a curve of a complex shape instead of a real straight line. The experiment to measure the dependence of the audible pitch on the frequency of the tonal signal was so difficult for the subjects and researchers that no further experiments were carried out. 


\subsection{The Ratio of the Audible Pitch to the Sequence Number of the IHC in PMVM}

The graph in Figure 2 allows us to determine the audible pitch in the perception of a tone signal with any frequency.

Table 3. The ratio of the audible pitch Zmel to the sequence number NO of the IHC in PMVM.

\begin{tabular}{|c|c|c|c|c|c|c|c|}
\hline $\mathbf{F}, \mathbf{H z}$ & 450 & 500 & 630 & 794 & 1000 & 1260 & 1587 \\
\hline $\mathrm{Z}$, mel & 450 & 500 & 624 & 734 & 850 & 1011 & 1159 \\
\hline N0 & 657 & 718 & 870 & 1047 & 1241 & 1453 & 1680 \\
\hline K & 0,68 & 0,69 & 0,72 & 0,70 & 0,68 & 0,69 & 0,69 \\
\hline $\mathbf{F}, \mathbf{H z}$ & 2000 & 2520 & 3174 & 4000 & 5040 & 6348 & 8000 \\
\hline $\mathrm{Z}, \mathrm{mel}$ & 1293 & 1451 & 1613 & 1750 & 1869 & 2003 & 2134 \\
\hline N0 & 1898 & 2117 & 2323 & 2540 & 2738 & 2916 & 3092 \\
\hline K & 0,68 & 0,68 & 0,69 & 0,69 & 0,68 & 0,68 & 0,69 \\
\hline
\end{tabular}

Table 3 shows several frequencies with the audible pitch "Z, mel", sequence numbers "N0" for IHC, located in the middle of the general group of cells with increasing RP, and the ratio $\mathrm{K}=\mathrm{Z} / \mathrm{N} 0$. The ratio $\mathrm{Zmel} / \mathrm{N} 0=(0.70 \pm 0.02)$ in the frequency range from $450 \mathrm{~Hz}$ to $8000 \mathrm{~Hz}$. This means that the sequence number of the IHC, located in the middle of the general group of cells with increased RP, is a real physical parameter by which the auditory system remembers information about the frequency of the audio signal.

The auditory system stores information about the frequency of the sound signal based on it. Each frequency of the sound signal corresponds to a point on the main membrane of the cochlea with the highest amplitude of transverse oscillations. Its linear coordinate Lapex, relative to apex of the cochlea, depends on the frequency of the sound signal.

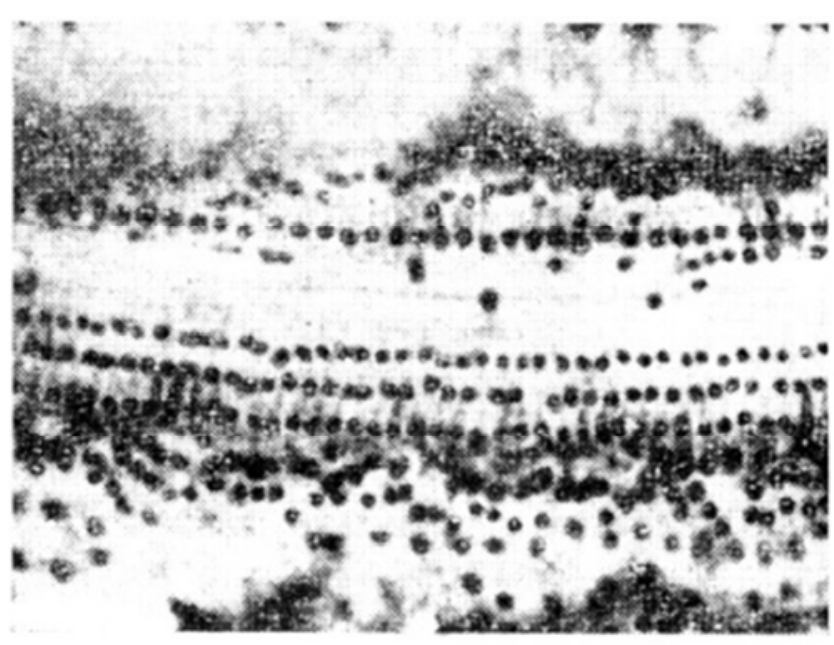

Figure 3. The organ of Corti of the inner ear of a guinea-pig [7].

The cells situated in a single row are inner hair cells (IHC); the cells in three parallel rows are outer hair cells (OHC). A single row of linearly arranged IHC can be considered as divisions of the measuring scale.

\subsection{Analysis of Frequency Threshold Curves of the Fibers of the Cochlear Nerve}

Figure 4 shows the FTC for 3 fibers of the cochlear nerve with characteristic frequencies $(\mathrm{CF}) 0.5 ; 1.0$ and $2.2 \mathrm{kHz}$ [8].
Each IHC is innervated by several of the spiral ganglion cells (SGC) with different excitation thresholds [8].

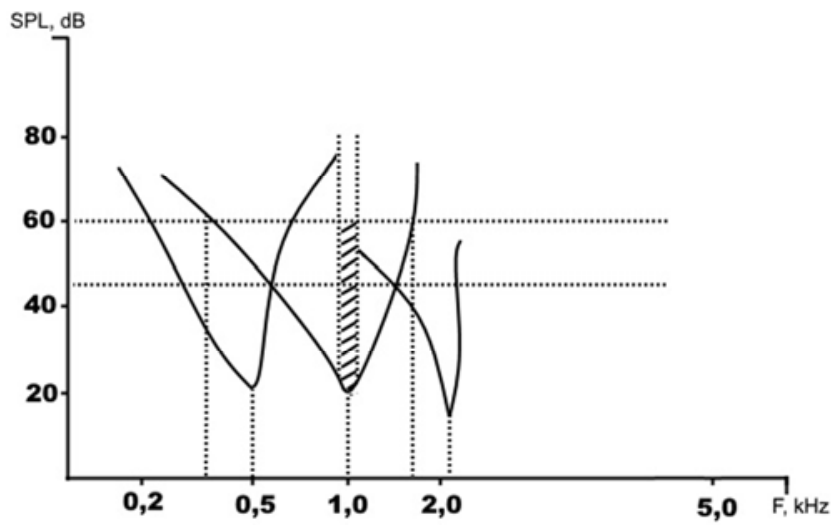

Figure 4. Frequency threshold curve (FTC) for three ANF [8].

With an increase in the RP of the innervated IHC, impulses are generated in the spiral ganglion cells (SGC). They are transmitted to the CCN via the AN fibers. The total discharge rate of all AN fibers that innervate a single IHC is proportional to the RP size of the innervated cell.

The abscissa axis is the tone frequency in $\mathrm{kHz}$, and the ordinate axis is SPL in $\mathrm{dB}$. The shaded $160 \mathrm{~Hz}$ wide band is the width of the frequency channel (FC) around PMVM for a frequency of $1 \mathrm{kHz}$. The points of intersection of the FTC side branches allow us to determine that after the perception of a tonal sound signal with a $1 \mathrm{kHz}$ frequency and $60 \mathrm{~dB}$ signal level the RP increases in all IHC with $\mathrm{CF}$ ranging from $0.5 \mathrm{kHz}$ to $2 \mathrm{kHz}$. Figure 5 shows a group of IHC with an increasing RP when perceiving a tone signal of medium intensity. The width of the FC around $1 \mathrm{kHz}$ frequency is 160 $\mathrm{Hz}$. The discharge rate of the IHC with a $\mathrm{CF}$ of $1 \mathrm{kHz}$ barely alters with a change in the frequency of the audible sound within the limits of the FC in the range from $920 \mathrm{~Hz}$ to 1080 $\mathrm{Hz}$. The distance between the IHC with $\mathrm{CF}=0.5 \mathrm{kHz}$ and $\mathrm{CF}$ $=2 \mathrm{kHz}$ is approximately $10 \mathrm{~mm}$ (see Figure 1). Approximately 1100 inner hair cells are located there. The IHC receptor potential increases to a maximum value of about $8 \mathrm{mV}$ at a signal level of $70 \mathrm{~dB}$. A further increase in signal level leads to the quantity of IHC with the highest RP gradually increasing to 146 cells, which triggers the pain threshold. The shape of the envelope curve changes to 
trapezoidal. In the process of memorizing information about the frequency and loudness of the audible tone, the human auditory system analyzes the degree of excitation of 146 IHC, forming one frequency channel (FC) [3].

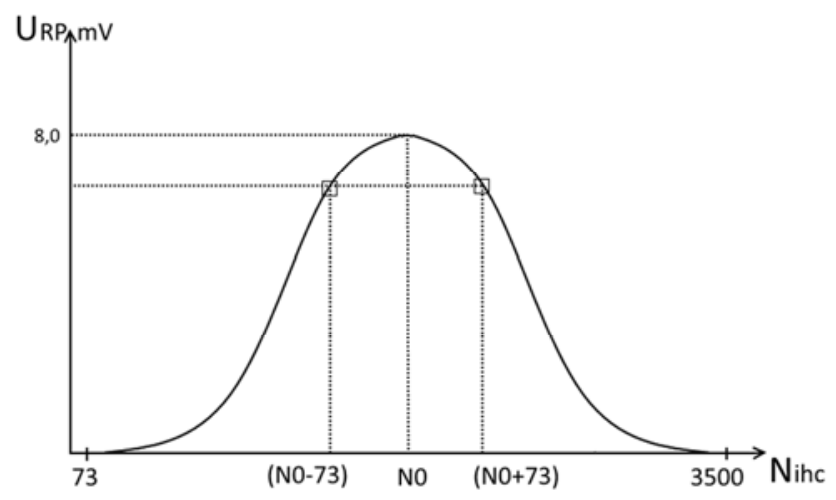

Figure 5. Envelope group of IHC with increased RP.

The abscissa axis shows sequence numbers of the IHC; the ordinate axis - the receptor potential of the excited IHC; the curved line is the envelope of the general IHC group with an increased RP.

The distance between the IHC with $\mathrm{CF}=0.5 \mathrm{kHz}$ and $\mathrm{CF}=$ $2 \mathrm{kHz}$ is approximately $10 \mathrm{~mm}$ (see Figure 1). Approximately 1100 inner hair cells (IHC) are located there. The IHC receptor potential increases to a maximum value of about $8 \mathrm{mV}$ at a signal level of $70 \mathrm{~dB}$. A further increase in signal level leads to the quantity of IHC with the highest RP gradually increasing to 146 cells, which triggers the pain threshold. The shape of the envelope curve changes to trapezoidal. Each IHC is innervated by several spiral ganglion cells (SGC) with different excitation thresholds, in which the RP is converted into a pulse sequence. SGCs actually work as an analog-to-digital converter. The total impulse density of all SGCs that innervate one IHC is proportional to the RP. From the SGC outputs, the pulses are transmitted along the fibers of the auditory nerve (AN) into the $\mathrm{CCN}$ to the 1-st projection of the frequency scale of the cochlea (PFSC). For example, the IHC with the sequence number N0 $=1240$ is in the middle of the general IHC group with an increased RP during the perception of a sound signal with a $1000 \mathrm{~Hz}$ frequency. When the frequency of the signal decreases, the whole group of IHC with an increased RP displaces along the BM towards the apex of cochlea. When the frequency increases, it moves towards the base of cochlea. The sequence number of the central IHC with an increased RP changes accordingly. For the auditory system, a change in the numerical value "NO" is a signal for sensing a change in the frequency of the audible sound.

When a sound signal is perceived, the auditory system locates the auditory neurons with sequence numbers (N0-73), N0, (N0 + 73) on the projection of FSC and transmits information about the numerical values of the sequence numbers to the long-term memory block. If the audible loudness changes, the discharge rate in the auditory neurons with the sequence number (N0-73) and $(\mathrm{N} 0+73)$ either decreases or increases subsequently. If the audible pitch changes, then the whole group of auditory neurons with an increased discharge rate shifts along the FSC projection and the discharge rate in the neurons with the sequence numbers (N0-73) and (N0 + 73) changes in different directions. The auditory system keeps the impulses of these neurons under control. A multidirectional change in the impulse density is a signal to start a new cycle of determining and storing information about the audible pitch.

\section{The Displacement of PMVM Point in the Perception of Tonal Signals with Different Frequencies}

The FTC of the 3 cochlear nerve fibers (CNF) shown in Figure 4, indicate that in the complex of elements OHC-BMIHC there are no conditions for distinguishing each individual frequency channel from 146 cells placed symmetrically with respect to the central IHC in the general group of cells with increased RP. The normalized point PMVM displacement along the BM during the change of the audio signal frequency, which is one of the most important operations, is performed here. What mechanism can solve this problem? According to Helmholtz [5], this may be a chain of resonant circuits connected in a series. Helmholtz's idea had no support among psychoacoustics specialists. Most experts believe that at the BM level there are bandpass filters (BF), which form 24 frequency channels.

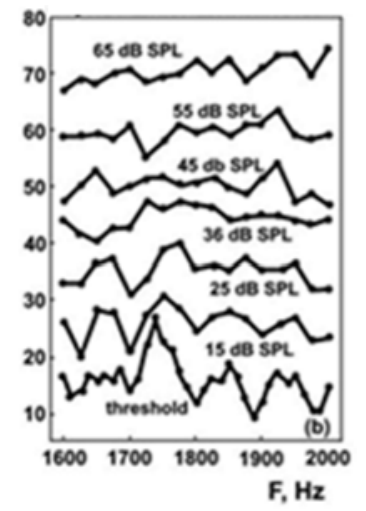

Figure 6. The fine structure of hearing threshold and loudness perception with the signal frequency range from $1600 \mathrm{~Hz}$ to $2000 \mathrm{~Hz}$ [9].

The alternation of maxima and minima suggests that the biophysical characteristics of the components complex OHCBM-IHC correspond to the frequency response of the chain of series-connected parallel resonant circuits. Through a series of parallel resonant circuits, a sound wave of any frequency can spread along the BM from the base to the top. At the point on the BM, where the frequency of the wave is closest to the resonance frequency of the circuit, the maximum vibration of the BM will be observed. The distance between the PMVM for frequencies in the range of $1600 \mathrm{~Hz}$ and $2000 \mathrm{~Hz}$ is approximately $2 \mathrm{~mm}$. On a $2 \mathrm{~mm}$ segment of the $\mathrm{BM}$ there are 5 resonant circuits (RC). One contour (circuit) is located along a piece of the BM with a length of $0,4 \mathrm{~mm}$. The number of these RCs located along the entire 32 
mm length of the BM depends on the results of experiments on the structure of auditory thresholds in the frequency range from $50 \mathrm{~Hz}$ to $8 \mathrm{kHz}$. With an increase in SPL of up to $60 \mathrm{~dB}$ above the threshold of audibility the amplitude of local irregularities on equal loudness curves is significantly reduced. There is a feasible explanation. There is a generator of spontaneous otoacoustic emission (GSOE) in the auditory system. In silence, the generator of spontaneous emission sends impulses to the OHC-BM-IHC complex via an afferent bundle of the fibers of the auditory nerve to maintain a weak oscillatory motion in the endolymph. As a result, a spontaneous discharge rate is recorded in silence in the cochlear fibers in the frequency range from approximately $500 \mathrm{~Hz}$ to $4 \mathrm{kHz}$. When you perceive a sound signal, the spontaneous action level (SAL) gradually decreases. When the SPL is over $70 \mathrm{~dB}$ the GSOA switches off and the curvature of equal volume lines noticeably decreases [10].

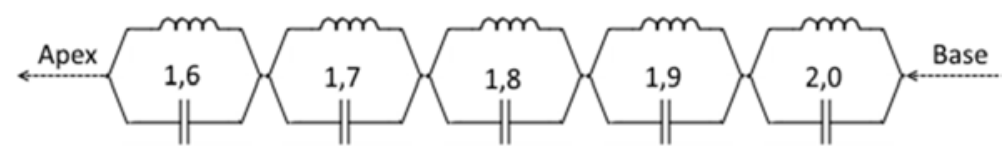

Figure 7. Resonant circuits.

Here is an interesting fact: in Figure 6, the largest peak amplitude is observed at a frequency of approximately 1750 $\mathrm{Hz}$. The frequency of $1760 \mathrm{~Hz}$ (musical note $A$ ) is the only frequency for which the PMVM is located exactly in the middle of the BM. The sequence number is calculated according to Table 3 of the IHC in the PMVM N0 $=1776$ (the discrepancy between the numbers 1760 and 1776 is less than $1 \%$ ). For $1600 \mathrm{~Hz}, \mathrm{~N} 0=1680$, for $2000 \mathrm{~Hz}, \mathrm{~N} 0=1900$. This means that 5 resonant circuits are located along the BM segment with a length of about $2 \mathrm{~mm}$, where 220 inner hair cells (IHC) are located. One resonant circuit is located along the BM segment with a length of about $0.4 \mathrm{~mm}$ (44 IHC).

The article [9] shows hearing thresholds for 9 subjects. The quantity of frequencies at which the subject hears the tone with the lowest intensity may be different. Of the 9 subjects, 3 had 4 minimums each, 2 had 5 minimums, and 4 subjects had $6,3,2$, and 1 minimums each. The quantity of minima depends on the individual hearing characteristics of the subjects, which influence the biophysical characteristics of the complex of elements OHC-BM-IHC.

The experiment on the exact measurement of hearing thresholds is very difficult for the subjects due to the change in the level of spontaneous activity with an increase in the intensity of the audible tone and its reverse effect. With an increase in SPL, the level of SA decreases, while a decrease in SPL is followed by an increase in level of SA. The process of changing the level of SA has some inertia. It is important to note that the experiment on the definition of hearing thresholds allows to determine the number of seriesconnected resonant circuits in different frequency ranges.

\section{The Correlation Between Difference Limens Frequency Resolution and the Duration of the Tone Audio Signal}

Figure 8 shows the results of an experiment to measure the dependence of the DLFs on the duration of the tone. Zwicker and Feldkeller were looking for a reason why the lines of the graph for 3 different frequencies are parallel. Quote: "Increasing any frequency by one Frequency Channel (FC) always leads to an increase in the audible pitch by $100 \mathrm{mel}$ " [3, p. 101].

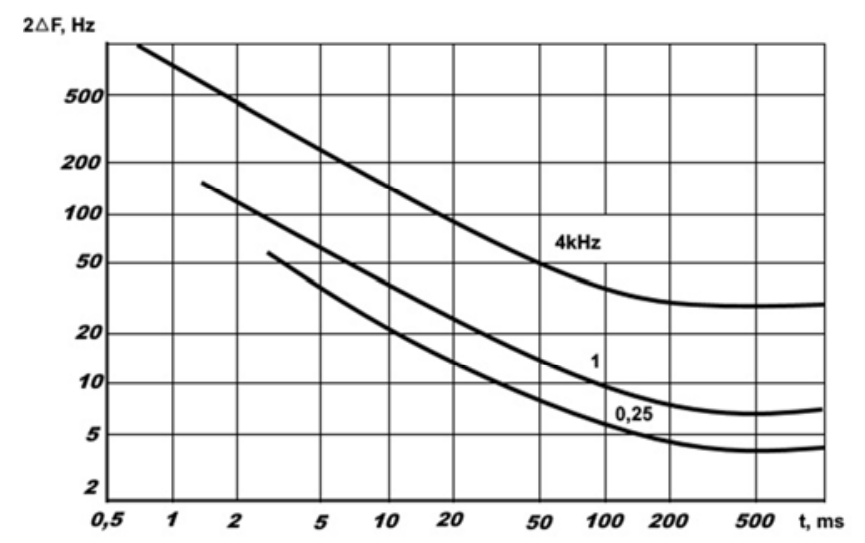

Figure 8. The correlation between the minimum perceived frequency interval between tonal pulses and the pulse duration [3].

In other words: a change in the audible pitch by $100 \mathrm{mel}$ means the displacement of the PMVM by 146 IHC along the BM. Table 4 shows the required PMVM shift to distinguish the change in frequency of 2 consecutive tones. The required PMVM shift depends only on the duration of the tone signal and does not depend on the signal frequency. There was one final step for Zwicker and Feldkeller to go from the conventional unit of measurement of the audible pitch " 1 mel" to the real physical parameter "the sequence number of the central IHC". They did not take it.

Other authors have attempted to carry out experiments to study the dependence of DLFs on the duration of the tone signal in the range from $20 \mathrm{~ms}$ to $200 \mathrm{~ms}$ [2, 11]. The results of these experiments confirm the significant dependence of the DLFs on the duration of the tone signal and a slight dependence on the signal frequency.

Table 4. The required PMVM shift to distinguish between changes in the frequency of 2 consecutive tones.

\begin{tabular}{llllllll}
\hline $\mathbf{F}, \mathbf{H z}$ & $\mathbf{t}, \mathbf{M c}$ & $\mathbf{2}$ & $\mathbf{5}$ & $\mathbf{1 0}$ & $\mathbf{2 0}$ & $\mathbf{5 0}$ & $\mathbf{1 0 0}$ \\
\hline \multirow{2}{*}{$\mathrm{F} 1=250 \mathrm{~Hz}$} & $2 \mathrm{x} \Delta \mathrm{F}, \mathrm{Hz}$ & 70 & 36 & 23 & 13,5 & 7,8 & 5,4 \\
& $\Delta \mathrm{N} 1$ & 100 & 51 & 33 & 19 & 4,2 \\
\hline
\end{tabular}




\begin{tabular}{lllllllll}
\hline F, Hz & t, $\mathbf{M c}$ & $\mathbf{2}$ & $\mathbf{5}$ & $\mathbf{1 0}$ & $\mathbf{2 0}$ & $\mathbf{5 0}$ & $\mathbf{1 0 0}$ & $\mathbf{2 0 0}$ \\
\hline \multirow{2}{*}{$\mathrm{F} 2=1000 \mathrm{~Hz}$} & $2 \mathrm{x} \Delta \mathrm{F}, \mathrm{Hz}$ & 105 & 58 & 39 & 23 & 11,6 & 9,1 & 6,7 \\
& $\Delta \mathrm{N} 2$ & 95 & 53 & 35 & 21 & 11 & 8 & 6 \\
$\mathrm{~F} 3=4000 \mathrm{~Hz}$ & $2 \mathrm{x} \Delta \mathrm{F}, \mathrm{Hz}$ & 500 & 232 & 169 & 91 & 50 & 36 & 31 \\
& $\Delta \mathrm{N} 3$ & 104 & 48 & 35 & 19 & 10 & 7 & 7 \\
& $\Delta \mathrm{N}$ ihc, mittel & 101 & 50 & 34 & 19 & 10 & 8 & 6 \\
\hline
\end{tabular}

Note: $(2 \mathrm{x} \Delta \mathrm{F}), \mathrm{Hz}$ is measured according to the graph in Figure 8 [3]; $\Delta$ Nihc - how the sequence number of the central IHC changes when it perceives the minimum perceived change in frequency; $\Delta$ Nihc, mittel - the average value of the minimum required PMVM shift for the sensation of changing the frequency of the tone for 3 frequencies.

\section{A Potential Way to Isolate a Group of 146 IHC with Increased RP}

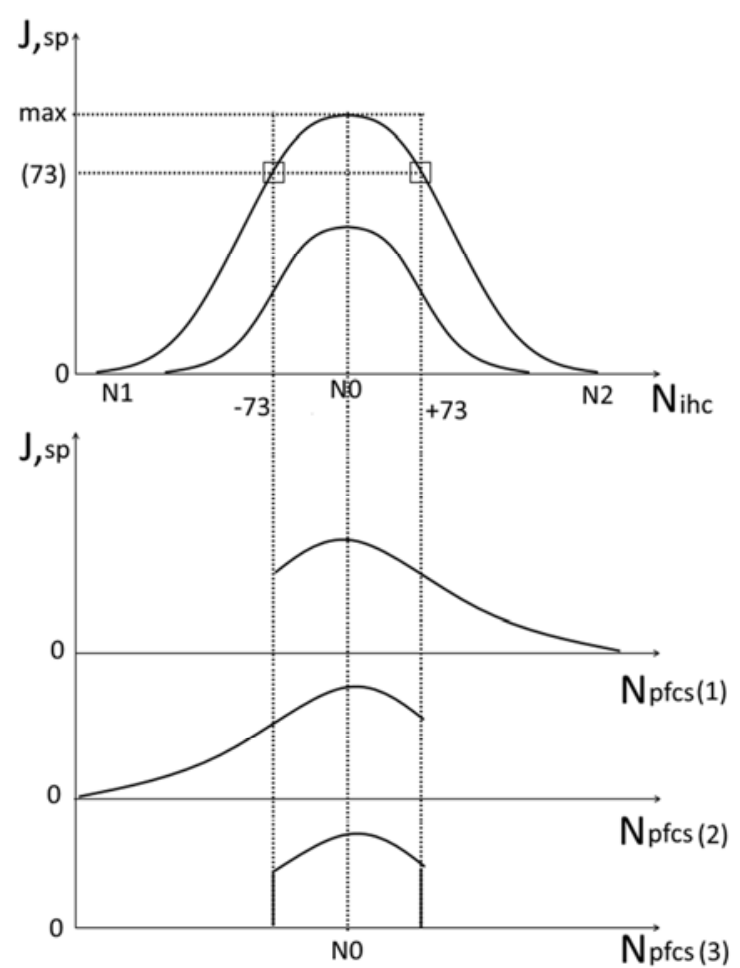

Figure 9. The selection of a 146 auditory neurons (AN) group, forming a frequency channel (FC) [10].

The abscissa shows the sequence numbers of the IHC (N ihc) and the auditory neurons (AN), which innervate the corresponding IHC; the ordinate axis is the impulse density $\mathrm{J}$, sp (imp. / s) in relative units for the auditory neurons that form the frequency channel scale (FCS). (N1 - N2) - a group of IHC with an increased RP at the perception of a tone signal with a SPL of $70 \mathrm{~dB}$ above the hearing threshold. N0 is the sequence number of the IHC located in the PMVM. There are 73 neurons between the vertical dashed lines along the Npfcs (1), Npfcs (2) and Npfcs (3) axes. [10].

The selection of a 146 central auditory neurons group is possible only if the number "146" is present in the algorithm for perceiving information about the frequency of a sound signal. It can be assumed that in a linearly located group of auditory neurons along the Npfsc (1) axis, the exciting synapse of each auditory neuron (AN) is connected to a neuron on the Nihc axis with a sequence number $(\mathrm{N})$, and the inhibitory synapse of this neuron is connected to a neuron with a sequence number $(\mathrm{N}+146)$. In a linearly located group of auditory neurons along the Npfsc (2) axis, the exciting synapse of each AN is connected to a neuron with a sequence number $(\mathrm{N})$ on the Nihc axis, and the inhibitory synapse is connected with a sequence number (N-146). A neuron can be considered as a digital "I" chip. Of all the neurons located along the Npfsc (3) axis, only neurons with sequence numbers from $(\mathrm{N} 0-73)$ to $(\mathrm{N} 0+73)$ will have an increased impulse density [10].

The scientific literature doesn't contain any assumptions on the practicable way of isolating 146 auditory neurons located in the middle of a general group of cells with a high discharge rate.

\section{Discussion}

\subsection{The Impossibility of Coding Frequency Information Using the Temporal Principle}

It is known that the discharge rate of a single AN fiber, when it perceives a tonal signal with a characteristic frequency and a duration of $400 \mathrm{~ms}$, increases from about 25 to 75 spikes per stimulus [12]. In this case, the duration of the interpulse interval decreases from $16 \mathrm{~ms}$ to $5 \mathrm{~ms}$. The discharge rate of a single AN fiber depends both on the intensity of the audible signal and, to a much lesser extent, on the change in the frequency of the tone signal within one frequency channel (see Figure 4). With a consistent perception of tone signals with a $920 \mathrm{~Hz}$ and $1080 \mathrm{~Hz}$ frequency, the period of the signal varies from $1.09 \mathrm{~ms}$ to $0.93 \mathrm{~ms}$, or $0.16 \mathrm{~ms}$. After summing up the discharge rate in individual AN fibers innervating one IHC at the synapses of the auditory neuron (AN) at the input of the 1-st PFCS in $\mathrm{CCN}$ [4], it is difficult to assume that the information on the change of the period of the sound signal by $0.16 \mathrm{~ms}$ can persist in the total pulse flow of the auditory neuron. At the same time, when the tone signal frequency changes and the PMVM shifts along the BM, the nominal frequency of the signal is the only parameter to compare the frequency of the sound wave and the frequency of the resonant circuit. In this case, the human DLFs depend on one real physical parameter: the change in the sequence number of the central IHC in the general group of cells with an increased RP.

Another convincing argument in favor of the assumption about coding information on the signal frequency according to the place principle is the study of auditory sensations when cochlear implant $(\mathrm{CI})$ users perceive a sound signal [13]. A 
flexible tube with platinum electrodes is placed along the basilar membrane BM during the cochlear implantation. In different types of CI there can be 12, 16, 20, or 22 electrodes. The distance between the electrodes is from $2.4 \mathrm{~mm}$ to 1.2 $\mathrm{mm}$. When tuning the CI, identical pulse patterns are received for each individual electrode. For example, when using a SPEAK type encoding strategy in CI, the duration of a stimulating pulse is $0.2 \mathrm{~ms}$, and in the ACE strategy it is $0.06 \mathrm{~ms}$. The interpulse interval is $0.6 \mathrm{~ms}$. The loudness of the audible sound depends on the amplitude of the stimulating pulses. Audible pitch depends only on the distance of the stimulated electrode relative to apex of the cochlea $[13,14]$. During the stimulation of electrodes, pulses with exactly the same parameters are transmitted through the fibers of the auditory nerve to $\mathrm{CCN}$ as with the natural perception of sound. The duration of the spike is approximately $1 \mathrm{~ms}$, the duration of the interpulse interval may be in the range of $40 \mathrm{~ms}$ to $15 \mathrm{~ms}$. The further from the apex of the cochlea the stimulated electrode is located, the higher the sound the user of the CI hears. When he perceives a tonal sound signal with a certain frequency, the user of the $\mathrm{CI}$ in most cases hears a sound with a different pitch than he heard before hearing loss. For example, user of CI hears a sound with a frequency of $440 \mathrm{~Hz}$. Under normal hearing, when perceiving a tone with a frequency of $440 \mathrm{~Hz}$, the PMVM was located about $5 \mathrm{~mm}$ from the apex cochlea. In a $\mathrm{CI}$, an electrode that is stimulated at a signal frequency of $440 \mathrm{~Hz}$ can be at different distances from the apex of the cochlea, for example, from $8 \mathrm{~mm}$ to $12 \mathrm{~mm}$, depending on the type of CI. The group of AN fibers different from what it used to be before hearing loss will have an increased the discharge rate. The user of the CI will hear a different audible pitch. This can be seen as another proof of the coding of frequency information by the place principle.

\subsection{The Value of Zwicker and Feldkeller's Experiments for Neurobiology}

The human brain stores information about the frequency of an audible sound signal for the sole purpose of recognizing a similar sound when repeated perception. The physical characteristics of the sound signal: the frequency in "Hz" and the intensity in "dB" the brain does not determine. For example, a child hears the note "A" with a frequency of $440 \mathrm{~Hz}$. Human sensation: the point on the basilar membrane of the cochlea where the greatest amplitude of transverse vibrations is felt is at a distance of approximately $5.8 \mathrm{~mm}$ from the apex of the cochlea. Then the child hears a sound with a frequency of 880 $\mathrm{Hz}$. Now the brain feels that the point on the basilar membrane of cochlea where the greatest amplitude of oscillations is observed is at a distance of about $10.8 \mathrm{~mm}$ from the apex. The brain does not receive any other information until the sequence number N0 of the inner hair cell located in the middle of the General group of cells with increased RP is transmitted to the long-term memory block. This information is quite sufficient for the child to recognize both of these sounds when repeated perception and even be able to reproduce these sounds with sufficient accuracy. When a child begins to learn music and learns that the 1-st sound is called the note "A" and has a frequency of $440 \mathrm{~Hz}$, only then the previously memorized sequence number N0 and the words "A" and $440 \mathrm{~Hz}$ are combined into a single memory block in the higher parts of the brain. The child also remembers the voice control code to be able to reproduce the heard sound.

The projections of receptor cells of all sensory systems are organized in the same way: this is an orderly arrangement of the columns of sensory neurons. On the projections of the frequency scale cochlea (FSC) in all parts of the auditory system, the columns of neurons are arranged linearly, and the frequency information is memorized by the sequence number of the IHC in the PMVM. On the projections of the visual field, the columns of neurons are located in the form of a square $1000 \times 1000$. When perceiving visual images, information about geometric dimensions is stored by the numerical values of the image control points coordinates relative to the $\mathrm{X}$ and $\mathrm{Y}$ axes [15]. Information about the basic parameters of smells or tastes is stored according to the location coordinates on the projection of sensory neurons that innervate the receptor cells with the highest RP. Without Zwicker and Feldkeller's experiments there would have been no evidence of the possibility of using receptor cell projections as a measuring scale for memorizing the numerical characteristics of the main parameters of sensory stimuli. The neural network remembers the main characteristics of sensory stimuli for the sole purpose of to identifying a similar stimulus when represented. The neural network does not define any parameters of the physical characteristics of the stimuli in $\mathrm{Hz}, \mathrm{mm}$ or degrees of slope of the lines. In the neural network of the brain, when sensory stimuli are perceived, numerical information about the main sensory parameters is transmitted to the long-term memory block as a number in binary code. A neuron can be in 2 conditions: generating impulses (1) or "silent" (0). A possible way to remember a number in binary code in the group of interneurons is explained in the article [16].

\section{Conclusion}

The article provides evidence of the possibility of storing information about the frequency of the sound signal on the "principle of place", which is based on the analysis of the results of 5 known experiments. The most significant is the experiment on the measurement the central and boundary frequencies for 24 frequency channels [3]. According to Table 1, it is possible to calculate the distance from the apex of the cochlea "Lapex" to the inner hair cell (IHC), which is located in the middle of the general group of IHC with increased RP when perceiving a tonal audio signal of any frequency. According to the graph in Figure 1 the distance of the same IHC to the base of the cochlea "Lbase" can be measured. The reliability of the results of these 2 experiments is demonstrated in Table 2 . In the frequency range from 0.1 $\mathrm{kHz}$ to $10 \mathrm{kHz}$ :

$$
\text { Lbm }=\text { Lbase }+ \text { Lapex }=(30.6 \pm 1.1) \mathrm{mm}
$$


Such a coincidence of the results of determining the length of the BM from the apex to the base of the cochlea, obtained by a complex analysis of the results of 2 completely different experiments, can be considered as proof of the reliability of the results of these experiments. In Figure 3 the dependence of the audible pitch in conventional units "mel" on the frequency of the signal $\mathrm{F}, \mathrm{Hz}$ is shown [3]. Table 3 shows the ratio of the audible pitch ( $\mathrm{Zmel}$ ) to the sequence number N0 of the IHC located in the middle of the general group of cells with increased RP. The ratio Zmel / N0 $=(0.70 \pm 0.02)$ in the frequency range from $450 \mathrm{~Hz}$ to $8000 \mathrm{~Hz}$. This means that the sequence number of the IHC, located in the middle of the general group of cells with increased RP, is a real physical parameter by which the auditory system remembers information about the frequency of the audio signal. If we consider the dependence of the audible pitch on the frequency of the audio signal [3] as a result of a separate experiment, it is impossible to understand the features of perception of information about frequency in the human auditory system. However, if we consider the ratio ( $\mathrm{Zmel} /$ $\mathrm{N} 0$ ), then the basic principle of the functioning of the auditory system is manifested: information about the sound frequency is stored by means of the sequence number of the IHC, located in the middle of the general group of cells with increased RP. Without Zwicker and Feldkeller's experiments, it would be completely impossible to suggest such an algorithm for the functioning of the auditory system.

The projections of receptor cells for all sensory systems are organized in the same way: these are ordered columns of sensory neurons. The method of storing information about the basic parameters of sensory stimuli for all systems is the same: memorizing the coordinates of the location on the projection of sensory neurons that Innervate receptor cells with the highest RP.

\section{References}

[1] Hearing: Psychophysics, Physiology, and Models (2018). Acta Acustica united with Acustica, 104: 741-747.

[2] B. C. J. Moore, S. M. A. Ernst (2012). Frequency difference limens at high frequencies: Evidence for a transition from a temporal to a place code. JASA, 132: 1542-1547.

[3] E. Zwicker, R. Feldkeller (1967). Das Ohr als Nachrichtenempfänger. Stuttgart: Hierzel-Verlag: 256 p.

[4] M. Bollerott, H. G. Despang, W. Kluge, A. Schwarz (1996). Softwaremodell der natürlichen Cochlea. Acustica, 82: 102113.

[5] S. S. Stevens., H. Davis, M. H. Lurie (1935). The localization of pitch perception on the basilar membrain. J. Gen. Psychol., (13): 297-315.
[6] J. C. Steinberg (1937). Position of stimulation in the cochlea by pure tones. JASA 8: 176-181.

[7] R. Feldkeller, E. Zwicker (1957). Das Ohr als Nachrichtenempfänger. Stuttgart: Hierzel-Verlag: 103 p.

[8] N. Y. S. Kiang, M. B. Sachs, W. T. Peake (1967). Shapes of tuning curves for single auditory nerve fibers. JASA, 42: 1341-1342.

[9] M. Mauermann (2004). Fine structure of hearing threshold and loudness perception. JASA, 116: 1066-1088.

[10] M. Stefanovich, V. Pudov (2013). Peculiarities of auditory sensations in electrode hearing aids. Neurophysiological mechanisms of the formation of auditory sensations in the perception of a sound signal. Saarbrucken: LAMBERT. $120 \mathrm{p}$.

[11] H. Fastl, A. Hesse (1984). Frequency discrimination for pure tones. ACUSTICA 56: 41-47.

[12] A. R. Palmer, E. F. Evans (1979). On the peripheral coding of the level of individual frequency components of complex sounds at high sound levels. Exp Brain Res. Suppl II: 19-26.

[13] A. E. Vandali, C. Sucher, D. J. Tsang, C. McKay (2005). Pitch ranking ability of cochlear implant recipients: A comparison of sound-processing strategies. JASA, 117 (5): 3126-3138.

[14] C. McKay, K. R. Henshall (2002). Frequency-to-electrode allocation and speech perception with cochlear implants. J ASA, 111: 1036-1043.

[15] M. Stefanovich (2015). The process of memorizing of numeric values of main characteristics of stimulus in human sensory systems. Neurocomputers: development, application, 11: $35-40$.

[16] M. Stefanovich (2017). A number in binary code as a possible way to store information about the frequency of an audio signal in the human auditory system. Materials of the allRussian conference. Kazan, p. 477-490.

\section{Biography}

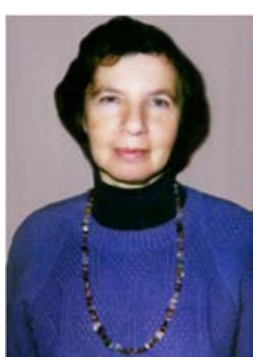

Margarita Stefanovich, I have worked 34 years as an engineer specializing in radio measuring instruments. I have several patents for inventions in the field of measuring technology. Now I am a pensionary and an independent researcher. Since 2000 I do my own research on hearing physiology and neurophysiology. Between 2012 and 2018, 9 of my articles and one my book were published: M. Stefanovich, V. Pudov "Peculiarities of auditory sensations in electrode hearing aids. Neurophysiological mechanisms of the formation of auditory sensations in the perception of a sound signal (2013)". Of particular interest to me is Cochlear Implantation. 